\title{
LIMITS ON FAST RADIO BURSTS FROM FOUR YEARS OF THE V-FASTR EXPERIMENT
}

\author{
S. Burke-Spolaor ${ }^{1,2,3}$, Cathryn M. Trotr ${ }^{4,7}$, Walter F. Brisken ${ }^{1,5}$, Adam T. Deller ${ }^{6}$, Walid A. Majid ${ }^{3}$, \\ Divya Palaniswamy ${ }^{4}$, David R. Thompson ${ }^{3}$, Steven J. Tingay ${ }^{4,7}$, Kiri L. WagstafF ${ }^{3}$, and Randall B. Wayth ${ }^{4,7}$ \\ ${ }^{1}$ NRAO, P.O. Box O, Socorro, NM 87801, USA; sspolaor@nrao.edu \\ ${ }^{2}$ California Institute of Technology, 1200 E. California Blvd., Pasadena, CA 91125, USA \\ ${ }^{3}$ Jet Propulsion Laboratory, California Institute of Technology, Pasadena, CA 91109, USA \\ ${ }_{5}^{4}$ ICRAR, Curtin University, Bentley, WA 6845, Australia \\ ${ }^{5}$ University of Minnesota, Minneapolis, MN 55155, USA \\ ${ }^{6}$ ASTRON, Oude Hoogeveensedijk 4, 7991 PD Dwingeloo, The Netherlands \\ Received 2015 June 24; revised 2016 May 18; accepted 2016 May 18; published 2016 August 2
}

\begin{abstract}
The V-FASTR experiment on the Very Long Baseline Array was designed to detect dispersed pulses of milliseconds in duration, such as fast radio bursts (FRBs). We use all V-FASTR data through 2015 February to report V-FASTR's upper limits on the rates of FRBs, and compare these with rederived rates from Parkes FRB detection experiments. V-FASTR's operation at $\lambda=20 \mathrm{~cm}$ allows direct comparison with the $20 \mathrm{~cm}$ Parkes rate, and we derive a power-law limit of $\gamma<-0.4$ (95\% confidence limit) on the index of FRB source counts, $N(>S) \propto S^{\gamma}$. Using the previously measured FRB rate and the unprecedented amount of survey time spent searching for FRBs at a large range of wavelengths $(0.3 \mathrm{~cm}>\lambda>90 \mathrm{~cm})$, we also place frequency-dependent limits on the spectral distribution of FRBs. The most constraining frequencies place two-point spectral index limits of $\alpha_{20 \mathrm{~cm}}^{4 \mathrm{~cm}}<5.8$ and $\alpha_{90 \mathrm{~cm}}^{20 \mathrm{~cm}}>-7.6$, where fluence $F \propto f^{\alpha}$ if we assume that the burst rate reported by Champion et al. of $R(F \sim 0.6 \mathrm{Jy} \mathrm{ms})=7 \times 10^{3} \mathrm{sky}^{-1} \mathrm{day}^{-1}$ is accurate (for bursts of $\sim 3 \mathrm{~ms}$ duration). This upper limit on $\alpha$ suggests that if FRBs are extragalactic but noncosmological, on average they are not experiencing excessive freefree absorption due to a medium with high optical depth (assuming temperature $\sim 8000 \mathrm{~K}$ ), which excessively inverts their low-frequency spectrum. This in turn implies that the dispersion of FRBs arises in either or both of the intergalactic medium or the host galaxy, rather than from the source itself.
\end{abstract}

Key words: pulsars: general - radio continuum: general

\section{INTRODUCTION}

In the past decade, we have gathered the first perspicuous evidence that there exists a large population of "fast radio bursts" (FRBs) with a potentially extragalactic origin (e.g., Lorimer et al. 2007; Thornton et al. 2013). However, uncertainties remain as to the origins of FRBs, and despite the large implied sky rate of $\sim 10^{4} \mathrm{sky}^{-1} \mathrm{day}^{-1}$, there have been only $\sim 20$ detections reported to date (Thornton et al. 2013; Burke-Spolaor \& Bannister 2014; Spitler et al. 2014; Champion et al. 2016; Petroff et al. 2015; Ravi et al. 2015), largely from the Parkes Radio Telescope. Other experiments have experienced nondetections that, so far, are consistent with the rate and sensitivity of detections (Siemion et al. 2012; Coenen et al. 2014; Law et al. 2014). Mounting evidence points to an extragalactic origin, namely, (1) the dispersion measures and rotation measures in excess of those expected from the Milky Way, and scattering timescales much smaller than those expected from a source of Galactic origin, imply that propagation through extragalactic media (from the intergalactic medium, a host galaxy, or the source itself) imparts these values (Kulkarni et al. 2014; Luan \& Goldreich 2014; Masui et al. 2015); and (2) the absence of FRB detections at low Galactic latitudes implies that "filtering" and signal dampening effects may be arising from propagation through the Milky Way (Burke-Spolaor \& Bannister 2014; Petroff et al. 2014). Recent detections and debate have added further complexity to the story: one FRB has been detected multiple times (Spitler et al. 2016). Another publication reported the first potential

\footnotetext{
${ }^{7}$ ARC Centre of Excellence for All-Sky Astrophysics (CAASTRO).
}

afterglow candidate to an FRB, implying that at least that particular FRB might be arriving from a galaxy at redshift $z=0.49$ (Keane et al. 2016), although whether the afterglow is truly associated with the FRB event is still under debate (Vedantham et al. 2016; Williams \& Berger 2016; Williams et al. 2016).

Since 2011, the V-FASTR experiment has run commensally on the Very Long Baseline Array (VLBA), which can provide a few tens of milliarcsecond localization of any detected FRB -hence the unique potential to localize an extragalactic pulse to a precise location within a host. The experiment has been described in detail in two publications (Thompson et al. 2011; Wayth et al. 2011), and the sensitivity of the experiment has been reported twice at earlier stages of observation (Wayth et al. 2012; Trott et al. 2013). In brief, V-FASTR uses an incoherent sum of the correlated data from the VLBA antennae to dedisperse and search for bursts in the resulting time series. For any promising candidate above our search threshold, we are able to access the baseband data and image the candidate to confirm it as an FRB. V-FASTR is one of a few FRB detection experiments currently operating worldwide at a broad range of frequencies, and thus has the potential to provide unique limits on average FRB population features such as spectral index and number count scaling. This is a particularly strong capability given the rapid and huge spectral index range reported for the repeating source by Spitler et al. (2016).

Since its campaign commenced, V-FASTR has not yet detected any FRBs. This paper provides a careful analysis of the sensitivity limits of the V-FASTR data taken to date and interprets the nondetection in terms of the physical parameters of FRBs with respect to previous FRB detection rates. 
Table 1

Observing Parameters for the V-FASTR Data as of 2015 February 24

\begin{tabular}{|c|c|c|c|c|c|c|}
\hline $\begin{array}{l}\text { Band } \\
(\mathrm{cm})\end{array}$ & $\begin{array}{c}\left\langle f_{\mathrm{ctr}}\right\rangle^{\mathrm{a}} \\
(\mathrm{GHz})\end{array}$ & $\begin{array}{c}\text { SEFD }^{b} \\
(\mathrm{Jy})\end{array}$ & $\begin{array}{c}\mathrm{SEFD}_{\mathrm{eff}} \\
(\mathrm{Jy})\end{array}$ & $\begin{array}{l}T_{\text {sys }}{ }^{\mathrm{c}} \\
(\mathrm{K})\end{array}$ & $\left\langle N_{\text {ant }}\right\rangle^{\mathrm{a}}$ & $\begin{array}{l}N_{\mathrm{hrs}} \\
(\mathrm{hr})\end{array}$ \\
\hline 90 & 0.318 & 2742 & 3439 & 184 & 9.2 & 24.2 \\
\hline 50 & 0.465 & 2744 & 3126 & 206 & 9.3 & 10.2 \\
\hline 20 & 1.550 & $302^{\mathrm{d}}$ & 311 & $31^{\mathrm{d}}$ & 9.4 & 1648.0 \\
\hline 13 & 2.278 & 347 & 357 & 30 & 9.0 & 82.8 \\
\hline $13 / 4^{\mathrm{e}}$ & 5.537 & 399 & 400 & 37 & 9.0 & 491.8 \\
\hline 6 & 5.949 & $244^{\mathrm{d}}$ & 245 & $28^{\mathrm{d}}$ & 9.1 & 1264.9 \\
\hline 4 & 8.418 & 327 & 327 & 36 & $10.1^{\mathrm{f}}$ & 1426.8 \\
\hline 2 & 15.082 & 543 & 543 & 67 & 9.6 & 790.0 \\
\hline 1 & 22.312 & $640^{g}$ & 640 & $68^{\mathrm{g}}$ & 9.6 & 1493.8 \\
\hline 0.7 & 43.161 & 1181 & 1181 & 106 & 9.3 & 797.7 \\
\hline 0.3 & 86.312 & 4236 & 4236 & 119 & 7.8 & 115.9 \\
\hline
\end{tabular}

Notes.

a Observing-time-weighted average.

${ }^{\mathrm{b}}$ https://science.nrao.edu/facilities/vlba/docs/manuals/oss/bands-perf accessed 2015 April 20. We assume a frequency-independent value for each band.

c Averaged across antennae, from the internal system measurements file on 2015 April 20. We assume an $f$-independent value for each band.

d Average of the two values reported for this receiver.

e V-FASTR treats the dichroic "S/X band" receiver observations as a single band when correcting for dispersion; here we quote the average values between this receiver's $\mathrm{S}$ and $\mathrm{X}$ bands.

${ }^{f}$ Some observations include non-VLBA antennae, e.g., the GBT, tied-array VLA, or EVN antennae. This occurs most frequently in the $4 \mathrm{~cm}$ band for geodesy experiments.

${ }^{\mathrm{g}}$ We use the values quoted for $1 \mathrm{~cm}$, which is closest to our $\left\langle f_{\mathrm{ctr}}\right\rangle$.

\section{FOUR YEARS OF V-FASTR}

\subsection{Data: 2011 April 24 to 2015 February 24}

As a commensal experiment, the observing setup of V-FASTR data is distinctively inhomogeneous. This is reflected by the values populating our five-dimensional observing parameter distribution, represented in Table 1 and Figure 1. The latter demonstrates that V-FASTR searches for FRBs at the full range of VLBA operating frequencies, and at a broad range of total bandwidth and channel width, both of which depend on the experiment on which V-FASTR is piggybacking. The range of time sample lengths reflects the fact that V-FASTR splits off a parallel data stream, for which the sampling time is optimized depending on the dispersion smearing at the given frequency setup of the primary experiment. Table 1 columns are as follows: (1) the observing receiver, as given by a representative wavelength; (2) the average center frequency of the V-FASTR data for that band; (3) the system equivalent flux density (SEFD) quoted for the VLBA; (4) the effective SEFD as described in Section 3.1; (5) the system temperature; (6) the average number of antennae used for the V-FASTR search; and (7) the cumulative number of hours spent using that receiver. Below we describe the dedicated V-FASTR observing program BT127, as well as the data removal heuristics we used to remove observing scans (from all observations) that are unsuitable for use in our limit analysis.

\subsection{BT127 Targeted Observing Program}

The one exception to V-FASTR's commensal operation has been a recent targeted program (BT127, which commenced in
2014) approved under the "VLBA Filler Project Challenge," in which projects can obtain large amounts of VLBA observation time in the gaps between long-duration observations. Such a filler mode is ideal for V-FASTR, as a list of high $(|b| \gtrsim 20)$ Galactic latitude targets can be defined at any right ascension (R.A.).

We constructed a list of 48 target fields spaced in R.A. by $0.5 \mathrm{hr}$. All fields lie north of the equator. Twenty-nine of these fields are at high Galactic latitudes $\left(|b|>20^{\circ}\right)$ and utilize observations that switch between the selected field and a highquality calibrator on a 10-minute cadence. For the remaining 19 R.A. for which a high Galactic latitude selection with nearby high-quality calibrator was not possible, we reverted to performing continuous observations of a field containing a strong calibrator near a declination of $+30^{\circ}$.

BT127 observations are scheduled for the object most appropriate for the time range covered by an available filler slot. BT127 observations utilize the full bandwidth available at $1.4 \mathrm{GHz}$ in order to achieve maximum sensitivity. As of 2015 February 24, a total of $\sim 350 \mathrm{hr}$ of observation have been obtained via BT127. However, not all of these data were processed by V-FASTR, and additional filtering of the data is described below. Therefore, $158 \mathrm{hr}$ of BT127 data have been usable for the analysis presented in this publication.

\subsection{Invalid Data}

From all data used by V-FASTR (including all projects, not just BT127), some were considered unusable due to the presence of a large number of spurious candidates-hence, the manual inspection step was not feasible or careful for these observations-or because the observing system had severe errors and did not record usable data. We thus do not consider data as follows:

1. Observations centered on either of the bright pulsars PSR J0332 +5434 or PSR J1136+1551.

2. Projects processed between 2014 January 30 and February 4 due to a communications error between the correlator and V-FASTR processing disk.

3. Projects processed between 2014 July 15 and 24 due to a temporary software error.

The invalid observations, in total encompassing $148 \mathrm{hr}(1.8 \%)$ of the V-FASTR data, are not considered in our analysis.

\section{LIMIT METHODOLOGY AND V-FASTR SENSITIVITY CONSIDERATIONS}

The limits derived in this paper are based on the framework of Trott et al. (2013), which takes into account the probabilistic nature of detection and transient event occurrence, enabling the construction of 2D probability distributions in the sensitivityrate parameter space. That work provides a full description of the framework's derivation, including an initial application of the framework specifically to the V-FASTR experiment. We provide here the relevant equations and alterations of their framework used for our analysis. Where elements are not explicitly expanded (e.g., $B(f, \theta)$ below), we refer the reader to Trott et al. (2013). The Trott et al. "detection performance metric," which casts sensitivity factors to a common reference

\footnotetext{
8 https://science.nrao.edu/enews/7.1/
} 

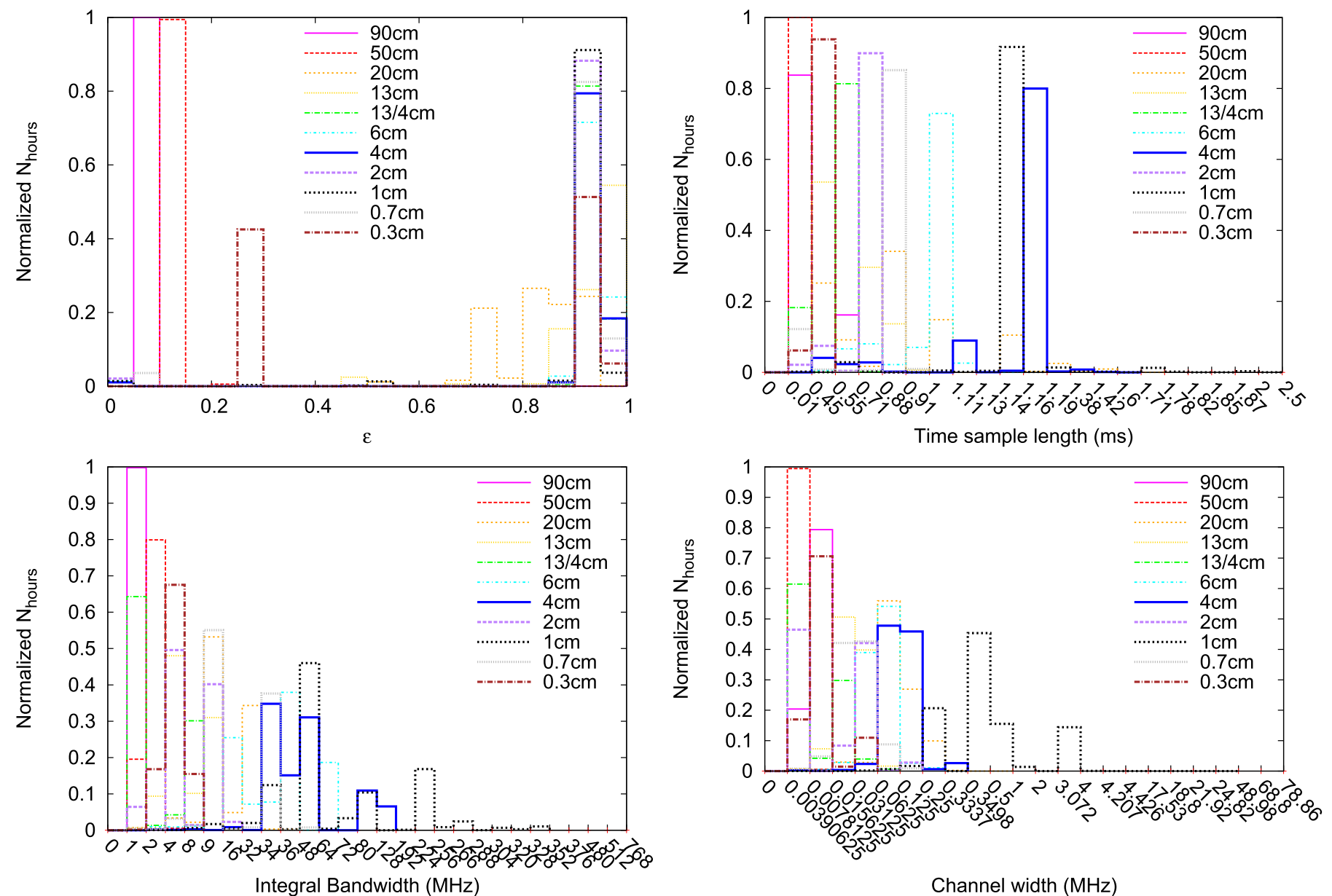

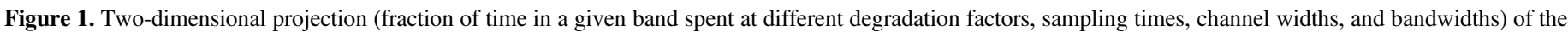

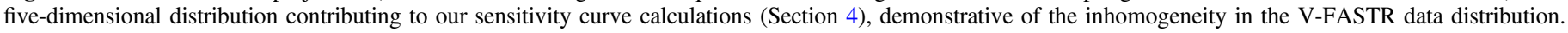

frequency $f_{0}$, is given by

$$
\epsilon S_{\text {actual }}\left(f_{0}, \theta\right) \sqrt{w}=\frac{C}{B(\theta)\left(\frac{f}{f_{0}}\right)^{\alpha}} \sqrt{\frac{S_{\text {sys }}^{2}(f) t_{\mathrm{s}}}{N_{\text {ant }}}} .
$$

Here $S_{\text {actual }}$ and $w$ are the actual source flux density and intrinsic pulse width, unobstructed by scattering and any observing instrumentation. Our detection degradation factor $\epsilon$ differs from that given by Trott et al. and is described in Section 3.3. The factors $t_{\mathrm{s}}, C$, and $N_{\text {ant }}$ give the sampling time, the signal-tonoise detection threshold (a dimensionless multiplier of the noise), and the number of antennas summed for detection, respectively. $S_{\mathrm{sys}}$ is the frequency-dependent system sensitivity; while it varies for different receivers, we use a fixed value for

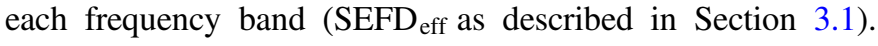
$B(\theta)$ is the antenna pattern. The transient signal itself might be frequency dependent, represented here by the power-law spectral index $\alpha$.

A source with true flux density $S_{\text {actual }}$ is measured with a flux density that includes statistical noise, which is Gaussiandistributed with mean $\mu=S_{\text {actual }}$ and data variance $\sigma^{2}$. The probability that a source of flux density $S_{\text {actual }}$ will be detected above a threshold given by $C \sigma$ is given by the cumulative distribution function:

$$
\begin{aligned}
P\left(S_{\text {actual }}>C \sigma\right) & =\int_{C \sigma}^{\infty} \mathcal{N}\left(S_{\text {actual }}, \sigma^{2}\right) d S \\
& =\frac{1}{2}+\frac{1}{2} \operatorname{erf}\left(\frac{S_{\text {actual }}-C \sigma}{\sqrt{(2) \sigma}}\right),
\end{aligned}
$$

where $\mathcal{N}\left(\mu, \sigma^{2}\right)$ denotes a Gaussian distribution. The cumulative probability in the rightmost part of the equation describes the exclusion regions. The cumulative probability that an event is not detected (due to noise) is the complementary function, $1-P\left(S_{\text {actual }}>C \sigma\right)$ (Trott et al. 2013).

The 2D probability distributions are formulated as a test of the alternate hypothesis, $1-P_{\text {null }}$, where the null hypothesis is that at least one source would have been detected when $n$ events have occurred:

$$
P_{\text {null }}=\sum_{n>1}\left(\left[1-P\left(S_{\text {actual }}<C \sigma\right)^{n}\right] \times Q(n ; \lambda)\right) .
$$

Here, $Q(n ; \lambda)$ is a Poisson distribution, $\lambda /\left(\mathrm{FOV} \times \mathcal{T}_{\text {obs }}\right)$ is the expected number density of events per field of view and total observing time, and $C \sigma$ refers to a detection threshold of $C$ times the noise, $\sigma$. The alternate hypothesis is the probability that events that occurred were not detectable by V-FASTR. The 
net probability for $N$ independent experiments is

$$
P_{\mathrm{tot}}=\prod_{i=1}^{N}\left[1-P_{\mathrm{null}}, i\right] .
$$

In this way, any number of inhomogeneous experiments can be combined into a single constraint on the rates of millisecond transients in the phase space of $\left(\epsilon S_{\text {actual }} \sqrt{w}, \mathrm{FOV} \times T_{\mathrm{obs}}\right)$, which represent the true flux and the true rate of bursts.

To summarize this process for clarity: in order to compute Equation (4) for a given instrumental setup, we use the observational parameters represented in Figure 1 to calculate the sensitivity thresholds for Equation (2) as given by Equation (1). Each instrumental setup has a given field of view and on-sky time, and these are used to calculate $Q(n ; \lambda)$ in Equation (3) above. We then use Equation (4) to combine the probabilities from various instrumental setups into the probability that V-FASTR would have detected a burst of a given true flux $S_{\text {actual }} \sqrt{w}$ and true rate $\mathrm{FOV} \times T_{\text {obs }}$.

\subsection{SEFD and Sky Temperature}

In our analysis, we take a band-averaged system sensitivity $S_{\text {sys }}$ to be the effective system equivalent flux density ( $S E F D_{\text {eff }}$ ) for the VLBA. The quoted SEFD of the VLBA is reported in Table 1, and we estimate $\mathrm{SEFD}_{\text {eff }}$ as reported below. The SEFD and $T_{\text {sys }}$ values reported in Table 1 correspond to a fairly optimistic observing configuration and may be further affected by telescope elevation, atmospheric sky temperature, and interference. Sensitivity values in typical observations can be on average $<20 \%$ worse at the lowest and highest frequencies, and roughly equal to those reported in this table for intermediate $\mathrm{GHz}$ frequencies (the most stable $T_{\text {sys }}$ values). The effects of harmful interference on the SEFD are mitigated by its automatic removal performed in the V-FASTR pipeline.

Notable amounts of time are spent observing the Galactic plane, at which-particularly at lower frequency-the background sky temperature can be comparable to the receiver temperature and therefore represent a significant loss in sensitivity. Thus, the effective SEFD for a pointing is a skyposition- and frequency-dependent scaling of the SEFD:

$$
\operatorname{SEFD}_{\text {eff }}(f, l, b)=\operatorname{SEFD} \cdot \frac{T_{\text {sky }}(f, l, b)+T_{\text {sys }}}{T_{\text {sys }}} .
$$

We determined $T_{\text {sky }}$ from the observation-based model of de Oliveira-Costa et al. (2008). A representative $T_{\text {sky }}$ for an observation is taken as the observing-time-weighted average of $T_{\text {sky }}(l, b)$ at the highest and lowest observed frequencies. It is clear that for V-FASTR observations to date, background sky temperature is only a notable factor at the $2 \mathrm{GHz}$ band and lower.

\subsection{The V-FASTR Detection Threshold}

The signal-to-noise detection threshold value, $C$, is nominally defined as a multiplication over the thermal noise in the receiver; however, due to the presence of radio frequency interference (RFI), $C$ can represent a larger sensitivity value. For millisecond pulse searches, typical values range from $C=6$ on the low end to $C=10$ on the high end (e.g., McLaughlin et al. 2006; Thornton et al. 2013; Burke-Spolaor \& Bannister 2014; Spitler et al. 2014). The impact of RFI on $C$ has, to our knowledge, not yet been considered in any FRB sensitivity analysis for single-dish experiments. V-FASTR has some intrinsic RFI advantage over single-dish experiments, as the spatially disparate antennae form a natural anti-coincidence filter (e.g., Thompson et al. 2011). The V-FASTR processing pipeline also performs adaptive RFI excision of narrow- and broadband signals as described in Wayth et al. (2012), which removes the bulk of potent RFI. Severely sensitivity-impacting narrow- and broadband signals have been seen at the manual inspection stage in only a negligible fraction of the newer broadband data.

V-FASTR is unique in that it incorporates multiple stations, each of which suffers different location-dependent RFI. It accounts for this dynamically by a pulse injection and detection system (Thompson et al. 2011), a robust estimator that sums signals while excising one or more extreme stations at each time step. The resulting time series is closer to Gaussian. Its noise is estimated from the data, and thresholds are adapted periodically to maintain a $7 \sigma$ cutoff. However, as our dynamic threshold is not tracked, we cannot use this information to precisely determine $C$ for V-FASTR. We therefore use the conservative estimate of $C=10$ for our limit analysis below.

\subsection{Instrumental Broadening and Scattering}

Several pulse broadening effects can reduce the detected signal strength. These interplay between natural broadening effects (i.e., scattering in the interstellar and intergalactic medium) and instrumental effects. The detected pulse width of an intrinsically unresolved pulse will be the square sum of the intrinsic pulse width and several broadening effects:

$$
t_{\mathrm{eff}}^{2}=w^{2}+t_{\mathrm{s}}^{2}+\tau^{2}+\left(\frac{k \mathrm{DM} \Delta f}{f^{3}}\right)^{2},
$$

where the final term is the "dispersion broadening" from a dispersion within finite channel bandwidth $\Delta f$ at frequency $f$, both in $\mathrm{MHz}$, with constant $k=8.3 \times 10^{3} \mathrm{MHz} \mathrm{cm}^{3} \mathrm{pc}^{-1}$. DM refers to the dispersion measure in units of $\mathrm{pc} \mathrm{cm}^{-3}$. The scattering timescale is represented by $\tau$, which typically has a power-law frequency dependence; when combining observations taken at different frequency, as is required for our analysis, one can scale the scattering expected at a common reference frequency such that $\tau_{\mathrm{s}}=\tau_{0}\left(f / f_{0}\right)^{\mu}$. For pulsars in the Galaxy and for all scattered FRBs yet observed, $\mu \sim-4$. Note that we assume here that detection experiments will search at a variety of pulse widths so that their detection integration time $\simeq t_{\text {eff }}$.

The dispersion and scattering of a fast transient can dominate $t_{\text {eff }}$ and degrade observational sensitivity. Using the Trott et al. framework, we can define a degradation factor, $\epsilon$, which represents the loss from an optimal signal-to-noise ratio $(\mathrm{S} / \mathrm{N})$ due to broadening, given intrinsic width $w$. Our $\epsilon$ encompasses all losses as reflected in Equation (6), not just scattering losses as in Trott et al. (2013). The ideal $\mathrm{S} / \mathrm{N}$ is

$$
\mathrm{S} / \mathrm{N}_{\mathrm{opt}} \propto S_{\text {intrinsic }} \sqrt{w} \text {. }
$$

When broadening occurs, systems typically find an optimized $\mathrm{S} / \mathrm{N}$ by integrating longer durations (up to a limit); however, this decreases both the noise and the signal at different rates ( $\sqrt{t}$ and $t$, respectively) such that the detected $\mathrm{S} / \mathrm{N}$ after 
broadening is

$$
\mathrm{S} / \mathrm{N}_{\text {broad }} \propto \frac{S_{\text {intrinsic }}}{t_{\text {eff }} / w} \sqrt{t_{\text {eff }}},
$$

and therefore the degradation factor is simply

$$
\epsilon \equiv \frac{\mathrm{S} / \mathrm{N}_{\mathrm{broad}}}{\mathrm{S} / \mathrm{N}_{\mathrm{opt}}}=\min \left(1, \sqrt{\frac{w}{t_{\mathrm{eff}}}}\right)
$$

Note that in the above equations we use $\sqrt{w}$ rather than $w$ because the $\mathrm{S} / \mathrm{N}$ scales as the square root of time. As in Trott et al. (2013), our use of $\mathrm{S} / \mathrm{N}$, rather than a fluence or flux density, allows us to assess the impact on $\mathrm{S} / \mathrm{N}$ of different experiments.

\subsection{Two Limit Scenarios for $\epsilon$}

We present the V-FASTR sensitivity in two ways:

1. Unknown properties of FRBs. We apply no assumption about FRB properties or Galactic/extragalactic broadening effects, so that the results can be compared in the future if FRB properties change with further detections. This equates to using a system with no degradation (i.e., $\epsilon=1$ ).

2. Extragalactic FRBs. Here we use the average properties of FRBs observed to date, plus a Galactic dispersion and scattering model and varying system setups, to determine $\epsilon$ for each V-FASTR observation. We attempt to correct for the observed latitude dependence of FRB rates (Burke-Spolaor \& Bannister 2014; Petroff et al. 2014) under the assumption that this dependence is due to propagation through the Galactic interstellar medium.

For the second approach, we use the NE2001 electron density model (Cordes \& Lazio 2002) to compute scattering and dispersion contributions from the Milky Way, as in BurkeSpolaor \& Bannister (2014). From the FRBs detected to date, we assume the following average values for this analysis:

$$
\begin{aligned}
\mathrm{DM}_{\text {exgal }} & =816 \mathrm{pc} \mathrm{cm}^{-3} \\
w_{\text {frb }} & =3 \mathrm{~ms} \\
\tau_{\text {exgal }} & =7 \mathrm{~ms} \text { at } 1.0 \mathrm{GHz} .
\end{aligned}
$$

Here the "exgal" subscript indicates that the quoted quantities exclude the contribution from electrons in the Milky Way itself, which must be added (in a sightline-dependent manner) to obtain the total observed dispersion and scattering. The values above come from 10 FRB DM exgal values: 330, 369, 521, 528, 675, 680, 710, 909, 1072, and $1553 \mathrm{pc} \mathrm{cm}^{-3}$; five FRBs with measured scattering and five with no scattering, projected to $1 \mathrm{GHz}: 3.7,5.3,17.7,17.7$, and $21.7 \mathrm{~ms}$; and five unscattered FRB widths after accounting for instrumental broadening: 0.57, 3.37, 0.69, 2.87, and $6.92 \mathrm{~ms}$ (Lorimer et al. 2007; Thornton 2013; Thornton et al. 2013; BurkeSpolaor \& Bannister 2014; Spitler et al. 2014; Petroff et al. 2015; Ravi et al. 2015). We thus determine $\epsilon$ for each
V-FASTR observation using the following formula:

$$
\begin{aligned}
\epsilon= & \sqrt{w_{\mathrm{frb}}}\left[t_{\mathrm{s}}^{2}+\tau_{\text {exgal }}^{2}(f)+\tau_{\mathrm{MW}}^{2}(f, l, b)\right. \\
& \left.+\left(\frac{k b\left(\mathrm{DM}_{\text {exgal }}+\mathrm{DM}_{\mathrm{MW}}(l, b)\right)}{f^{3}}\right)^{2}\right]^{-1 / 4},
\end{aligned}
$$

where again $\tau_{\text {exgal,Mw }}=\tau_{0}\left(f / f_{0}\right)^{\mu}$, and we use the NE2001 Milky Way electron density model to compute scattering and dispersion contributions from the Galaxy. We assume $\mu=-4$ in this analysis. The distribution of $\epsilon$ for each V-FASTR observing band is shown in the first panel of Figure 1.

\section{V-FASTR LIMITS ON FRBs AND MILLISECOND- DURATION RADIO TRANSIENTS}

We combine the inhomogeneous V-FASTR data set by forming a five-dimensional grid of observing band, time sample duration, channel bandwidth, integrated bandwidth, and degradation factor (a collapsed view of this is shown in Figure 1). Each of these grid cells contains the total on-sky time spent with that combination of observing parameters. The cells of this grid are treated as independent experiments, and the net probability (Equation (4)) for each limit plot in this section is formed by summing the probabilities over the cells of that band. It is this probability that is plotted in Figures 2-5.

Figures 3 and 5 show the most sensitive bands in $\epsilon S_{\text {actual }} \sqrt{w}-T_{\text {obs }} \times$ FOV space using this analysis considering our scenario without assumption of FRB properties. Figures 2 and 4 present the V-FASTR limits at the same bands using the "Extragalactic FRB" scenario (as described in Section 3.4).

\subsection{Comparison with Previous FRB Detection Rates}

Figure 2 compares the rate of several FRB detection experiments with the V-FASTR limits derived from $20 \mathrm{~cm}$ observations. We recalculated the FRB rates for these surveys to provide a consistent analysis with our limits, using values as reported in Table 2. The most constrained of these measurements come from Champion et al. (2016), who included the 10 FRBs detected from the HTRU-S Survey, and thus have the smallest Poisson error of the various experiments. This measurement is the most constrained because it reports the largest number of detections from a single survey with uniform parameters.

This figure demonstrates that our limit is currently consistent with all FRB measurements thus far; that is, we had a very low probability of a detection given the intrinsic FRB rates and flux densities implied by these experiments.

Nevertheless, our multidimensional limits in $S \sqrt{w_{\text {frb }}}$, $\mathcal{T}_{\text {obs }} \times \mathrm{FOV}$, and frequency space allow us to statistically constrain the physical parameters of the FRB population, in particular the average spectral index and the index of FRB source counts, as described below.

\subsection{FRB Spectral Index Limits}

We now consider physical limits on the observed FRB population to date. For the analysis in this and the next section, we take the $20 \mathrm{~cm}$ detections of Thornton et al. (2013, hereafter T13) and Champion et al. (2016, hereafter C16) as an "anchor point" for measured FRB rates. This is a simplifying assumption; further analyses have shown that the error bars and flux scale of FRB rates are highly uncertain and 


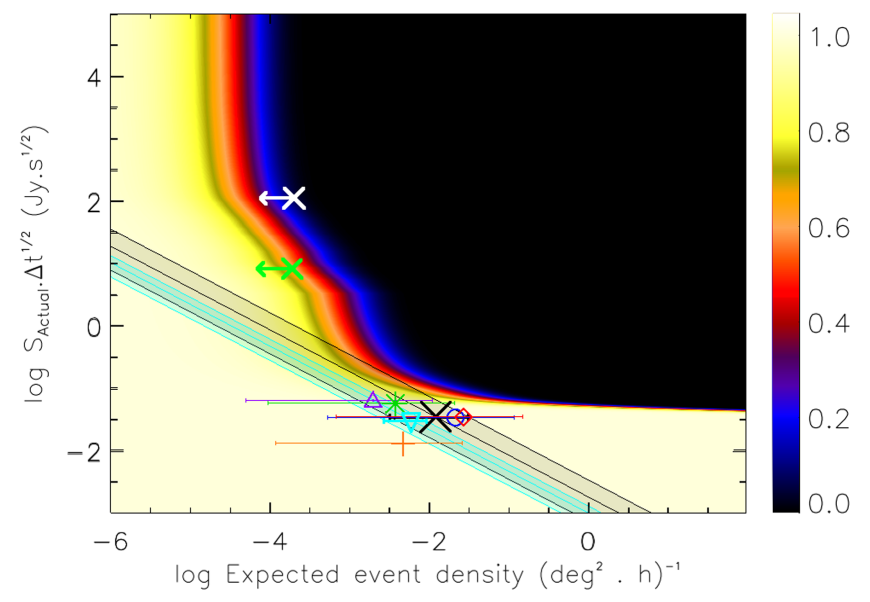

Figure 2. V-FASTR limits at $\lambda=20 \mathrm{~cm}$ using the "extragalactic FRB" scenario to derive $\epsilon$. The color bar represents the likelihood that V-FASTR would have seen zero events given the event rate and sensitivity metric. The points shown are as in Table 2: Lorimer et al. (2007)—green asterisk; T13black cross; Spitler et al. (2014) —orange plus sign; Burke-Spolaor \& Bannister (2014) - purple triangle; Petroff et al. (2015) — blue circle; Ravi et al. (2015) red diamond; Siemion et al. (2012) upper limit—green cross; Champion et al (2016) — cyan triangle. A standard $N(>S) \propto S^{-3 / 2}$ scaling is extrapolated from the T13 and Champion et al. measurements. The white cross indicates the V-FASTR limit that most constrains $\gamma$ (Section 4.3).

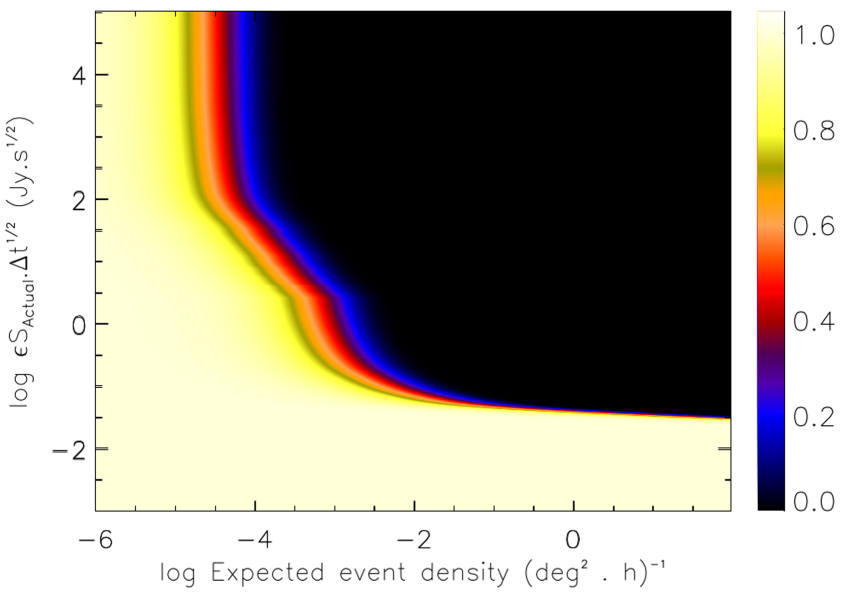

Figure 3. V-FASTR limits at $\lambda=20 \mathrm{~cm}$ without any assumptions for $\epsilon$.

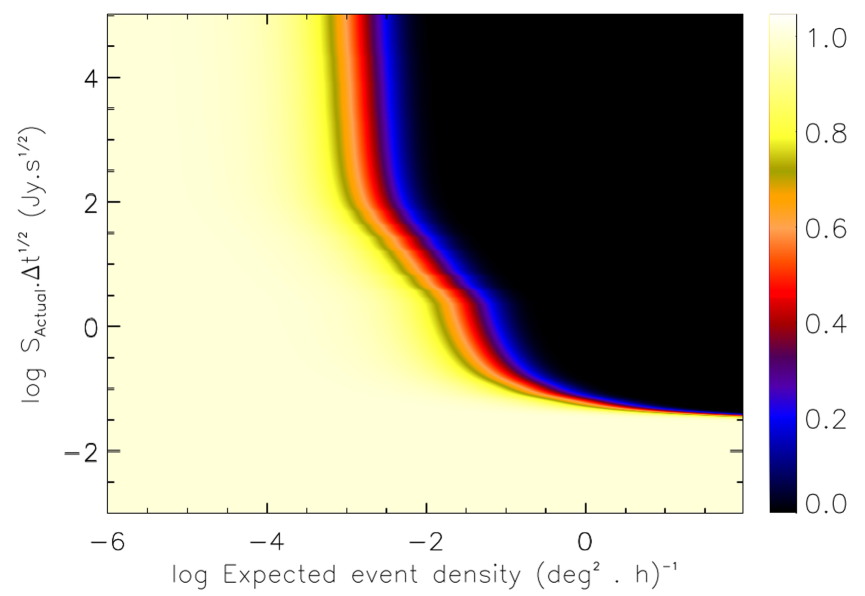

Figure 4. V-FASTR limits at $\lambda=4 \mathrm{~cm}$ using the "extragalactic FRB" scenario to derive $\epsilon$.

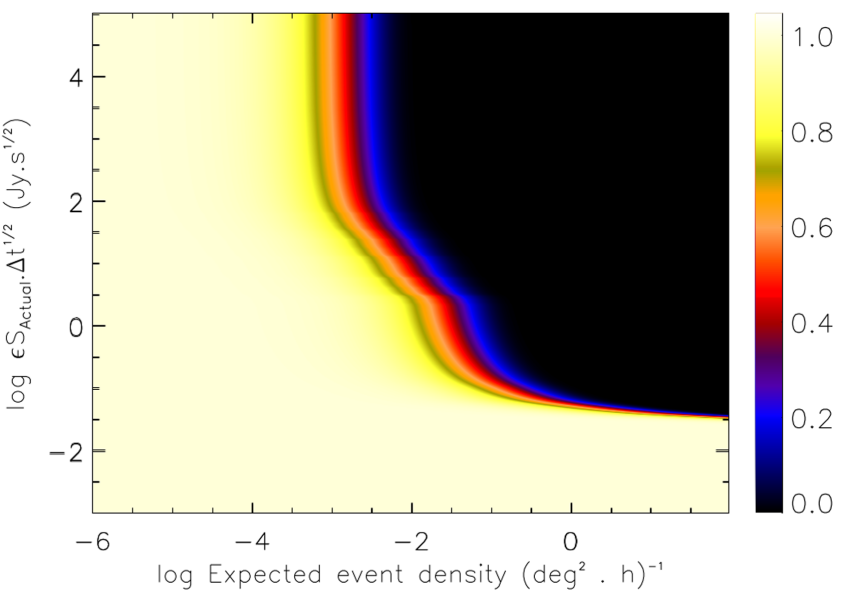

Figure 5. V-FASTR limits at $\lambda=4 \mathrm{~cm}$ without any assumptions for $\epsilon$.

Table 2

The Sensitivity Parameters of Surveys That Have Published FRB Discovery Rates, and for the "Fly's Eye" Experiment (Siemion et al. 2012), Which Searched for FRBs at $20 \mathrm{~cm}$

\begin{tabular}{lccccc}
\hline \hline References & $\begin{array}{c}\left\langle T_{\text {sky }}\right\rangle \\
(\mathrm{K})\end{array}$ & $\begin{array}{c}S_{\text {min,opt }} \\
(\mathrm{mJy})\end{array}$ & $\langle\epsilon\rangle$ & $\begin{array}{c}\mathcal{T}_{\text {obs }} \times \mathrm{FOV} \\
\left(\mathrm{hr} \mathrm{deg}^{2}\right)\end{array}$ & $N_{\text {FRB }}$ \\
\hline Lorimer et al. (2007) & 0.73 & 590 & 0.57 & 272.7 & 1 \\
Siemion et al. (2012) & 1.65 & $118 \times 10^{3}$ & 0.76 & 19992 & 0 \\
$\begin{array}{l}\text { Thornton et al. } \\
\quad(2013) \text { [T13] }\end{array}$ & 0.85 & 560 & 0.89 & 337.5 & 4 \\
$\begin{array}{l}\text { Spitler et al. (2014) } \\
\text { Burke-Spolaor \& }\end{array}$ & 1.31 & 210 & 0.90 & 215.2 & 1 \\
$\quad 1.70$ & 615 & 0.55 & 509.9 & 1 \\
$\quad$ Bannister (2014) & & & & & \\
$\begin{array}{l}\text { Retroff et al. (2015) } \\
\text { Chi et al. (2015) }\end{array}$ & 0.79 & 555 & 0.89 & 47.5 & 1 \\
$\quad 0.64$ & 555 & 0.88 & 38.0 & 1 \\
$\quad$ et al. (2016) & 0.92 & 560 & 0.88 & 1549 & 10 \\
\hline
\end{tabular}

Note. Sensitivity/Experimental parameters for most surveys are summarized in Burke-Spolaor \& Bannister (2014). We also use the Spitler et al. (2014) values assuming that their FRB detection was in the sidelobe of the Arecibo Antenna, with their reported $S_{\min }$ corrected for $T_{\text {sky }}$ and our $w_{\text {frb }}$. Apart from survey area, the Petroff et al. (2015) and Ravi et al. (2015) parameters are equivalent to those of T13. For consistent comparison with the V-FASTR limit results, we represent the lower limit of detectable intrinsic FRB flux density for an experiment as $S_{\mathrm{min}, \text { opt }}=C \cdot \mathrm{SEFD}_{\mathrm{eff}} / \sqrt{\mathrm{BW} \cdot w_{\mathrm{frb}}}$. The calculation uses the gain at the FWHM power point of the beam, in concordance with the $\mathcal{T}_{\text {obs }} \times$ FOV value reported here. Thus, in Figure 2 we plot $\left[S_{\text {min,opt }} \sqrt{w_{\text {frb }}} / \epsilon\right.$, $\left.N_{\mathrm{FRB}} /\left(\mathcal{T}_{\mathrm{obs}} \times \mathrm{FOV}\right)\right]$ for each experiment.

still the subject of ongoing debate (Keane \& Petroff 2015; Rane et al. 2016). Thus, while a maximum likelihood analysis would be the most rigorous path to derive the implied spectral dependence and number counts for FRBs, particularly if V-FASTR were to make a detection, such an analysis is not warranted in a null detection experiment with only two frequencies to fit. Note that T13 searched a subset of $\mathrm{C} 16$ data, and T13's reported rate is about double that of C16; we report results from both of these rates to demonstrate how our results change with different FRB rate measurements.

First, we consider limits on the spectrum of FRBs. We use our "extragalactic FRBs" scenario to place these limits, as these reflect most accurately any effects of frequency-dependent scattering and instrumental sensitivity. The most simplistic model for a radio spectral density is defined by power-law index $\alpha$, defined with fluence $F \propto f^{\alpha}$. We can place a two- 
Table 3

The Upper Limit on Power-law Spectral Index, $\alpha$, for Each of V-FASTR's Effective Center Frequencies Using the T13FRB Rate Measurement and the C16 Rate Measurements

\begin{tabular}{lccc}
\hline \hline $\begin{array}{l}\text { Band } \\
(\mathrm{cm})\end{array}$ & $\begin{array}{c}\left\langle f_{\mathrm{ctr}}\right\rangle \\
(\mathrm{GHz})\end{array}$ & $\begin{array}{c}\alpha(\mathrm{T} 13) \\
\text { limit }\end{array}$ & $\begin{array}{c}\alpha(\mathrm{C} 16) \\
\text { limit }\end{array}$ \\
\hline 90 & 0.318 & $>-6.4$ & $>-7.6$ \\
50 & 0.465 & $>-10.8$ & $>-11.5$ \\
20 & 1.55 & $<14.1$ & $<18.9$ \\
13 & 2.28 & $<24.1$ & $<33.1$ \\
6 & 5.95 & $<4.5$ & $<5.2$ \\
4 & 8.42 & $<4.0$ & $<5.8$ \\
\hline
\end{tabular}

Note. For wavelengths shorter than $4 \mathrm{~cm}$, The $\mathcal{T}_{\text {obs }} \times$ FOV product for those bands was too small to allow a comparison with the T13 rate.

point spectral limit on $\alpha$ at each of V-FASTR's single-receiver observing bands. We do this at the $20 \mathrm{~cm}$ event rate surface density of T13 (4/[337.5 $\left.\left.\mathrm{h} \mathrm{deg}^{2}\right]\right)$ and C16 (10/[1549 $\left.\left.\mathrm{h} \mathrm{deg}^{2}\right]\right)$, scaling $\alpha$ until our $5 \%$ probability point in $S \sqrt{w}$ meets that of $\mathrm{T} 13$. We use the $95 \%$ detection contour as a proxy for the 95\% limit on intrinsic source flux density, with the caveat that, in general, these are not the same quantity. This corresponds to the $95 \%$ confidence point in our null hypothesis, $P\left(H_{0}\right)$, that V-FASTR should have detected at least one burst given $\alpha$ and the T13 and C16 measurements. These frequency-dependent limits are shown in Table 3.

If FRB emission is truly broadband and has no break in its spectrum, we can state that our limits on spectral index are $-7.6<\alpha<5.8$ if the Champion et al. sensitivity is taken at face value. Specifically, our most stringent limits are for the 4 and $90 \mathrm{~cm}$ bands, such that $\alpha_{20 \mathrm{~cm}}^{4 \mathrm{~cm}}<+5.8$ and $\alpha_{90 \mathrm{~cm}}^{20 \mathrm{~cm}}>-7.6$. To our knowledge, the former is the first reported limit on the high-frequency spectral index (upper limit) of the FRB population as a whole. Recently, Karastergiou et al. (2015) also reported a two-point spectral index lower limit of $\alpha_{2 \mathrm{~m}}^{20 \mathrm{~cm}}>+0.1$.

The $\alpha$ values associated with known radio sourcesparticularly those with coherent emission as is expected in the case of FRBs - tend to be negative; however, for these our limits are not particularly constraining. For instance, while a few pulsars have flat spectra, they have declining spectra on average $\alpha \simeq-1.4$, and down to $\alpha \simeq-3.5$ (e.g., Bates et al. 2013).

Our limits, however, are interesting in light of two facts. First, one of the few reported FRBs with a spectral index measurement, FRB 121102, has been found to repeat and has erratic frequency-dependent amplitudes. These induce a huge range of indices measured for different detections, ranging from -10.4 to +13.6 (Scholz et al. 2016; Spitler et al. 2016). Our limits rule out that a significant population of bursts commonly have such extreme spectral indices.

Second, Kulkarni et al. (2014) discuss the effect of free-free absorption of FRB emission by a medium surrounding the FRB progenitor, which would also produce the excess DM observed in FRBs, that is, a noncosmological FRB scenario where most of the excess DM does not arise from the intergalactic medium. One of the implications of a medium with even a modest optical depth is that the spectrum will be strongly inverted, well above $\alpha \simeq+3.2$ for most bursts. Our upper limit on $\alpha$ thus implies that the average FRB population is not surrounded by compact, photoionized nebulae, assuming that the region remains optically thick up to at least $6 \mathrm{GHz}$. The implication for this is that the origin of the bulk of FRBs' excessive dispersion measures are on average not source-local if the source is $\mathcal{O}(10,000) \mathrm{K}$. Some FRB models pinpoint that FRBs arise in nearby galaxies; for these models to hold, therefore, the progenitor sources are implied to be preferentially positioned closer to the centers of the host galaxies, i.e., at a position where the host galaxy's interstellar medium can contribute the large excess DM observed in FRBs.

\subsection{FRB Source Count Limits}

Next, we can derive limits on a power-law scaling of FRB population number counts using our limits in the $20 \mathrm{~cm}$ band. Given our two-point $\alpha$ limits, we make the assumption that the small difference between the average V-FASTR and Parkes center frequencies-1.550 and $1.352 \mathrm{GHz}$, respectively-is negligible when we place our $\gamma$ limit (i.e., we assume $\alpha=0$ ). As with the spectral index, a typical dependence assumed for this scaling is a power law, $N(>S) \propto S^{\gamma}$. The standard $\gamma=-3 / 2$ projection is exhibited in Figure 2 for T13 and C16. Using V-FASTR's $20 \mathrm{~cm}$ limit, we can extrapolate the T13 and C16 measurements to our most sensitive 5\% confidence point, hence placing a limit of $\gamma<-0.5$ and $\gamma<-0.4$, respectively. As in the previous section, we use the $95 \%$ detection contour here as a proxy for the $95 \%$ limit on intrinsic source flux density, with the caveat that, in general, these are not the same quantity. We also include in Figure 2 the $20 \mathrm{~cm}$ measurement from the Allen Telescope Array's "Fly's Eye" experiment (Siemion et al. 2012), which provides the most constraining limit to date, of $\gamma<-0.76$.

Both $\gamma$ limits are in concordance with a cosmological population of FRBs in a Euclidean universe, where $\gamma=-1.5$. They also allow sufficient room, for instance, for a noncosmological population where our sensitivity is source limited rather than distance limited (e.g., Pen \& Connor 2015). It also still permits the possibility of luminosity or density evolution of FRBs over cosmic distances.

\section{CONCLUSIONS}

We have reported constraints on the FRB population based on observations using the V-FASTR experiment, which spans observing frequencies from $350 \mathrm{MHz}$ to $90 \mathrm{GHz}$. Analysis based on the nondetection of FRBs in V-FASTR data to date allows us to draw several conclusions:

1. Our nondetection at $20 \mathrm{~cm}$ is consistent with the FRB rate based on the detections of Champion et al. (2016). If FRBs are distributed with an $N(>S) \propto S^{\gamma}$ distribution with $\gamma=-1.5$, we find a probability of $\sim 91 \%$ that no FRBs would have been detected by V-FASTR data to date (Figure 2).

2. We have set multiwavelength constraints on the twopoint radio spectral index, $\alpha$. Our most stringent limits are for the 4 and $90 \mathrm{~cm}$ bands, such that $\alpha_{20 \mathrm{~cm}}^{4 \mathrm{~cm}}<+5.8$ and $\alpha_{90 \mathrm{~cm}}^{20 \mathrm{~cm}}>-7.6$. This in particular places constraints on any FRB-local photoionized nebula, which would, even with a modest optical depth, push spectral indices above $\alpha \sim 3$ (Kulkarni et al. 2014).

3. We place a limit on FRB number counts at $20 \mathrm{~cm}$ to be $\gamma<-0.4$. This limit, however, is not more constraining than the limits placed by other experiments on this parameter (Siemion et al. 2012). 
The National Radio Astronomy Observatory is a facility of the National Science Foundation operated under cooperative agreement by Associated Universities, Inc. We acknowledge the exellent commentary by two referees and the ApJ statistician on the paper's manuscripts. This work was carried out in part at the Jet Propulsion Laboratory, California Institute of Technology, under a contract with the National Aeronautics and Space Administration. Parts of this research were conducted by the Australian Research Council Centre of Excellence for All-sky Astrophysics (CAASTRO), through project number CE110001020. C.M.T. is supported by an Australian Research Council DECRA Fellowship through project number DE140100316.

\section{REFERENCES}

Bates, S. D., Lorimer, D. R., \& Verbiest, J. P. W. 2013, MNRAS, 431, 1352 Burke-Spolaor, S., \& Bannister, K. W. 2014, ApJ, 792, 19

Champion, D. J., Petroff, E., Kramer, M., et al. 2016, MNRAS, 460, 30

Coenen, T., van Leeuwen, J., Hessels, J., et al. 2014, A\&A, 570, A60

Cordes, J. M., \& Lazio, T. J. W. 2002, arXiv:0207156

de Oliveira-Costa, A., Tegmark, M., Gaensler, B. M., et al. 2008, MNRAS, 388,247

Karastergiou, A., Chennamangalam, J., Armour, W., et al. 2015, MNRAS, 452,1254
Keane, E. F., Johnston, S., Bhandari, S., et al. 2016, Natur, 530, 453

Keane, E. F., \& Petroff, E. 2015, MNRAS, 447, 2852

Kulkarni, S. R., Ofek, E. O., Neill, J. D., Zheng, Z., \& Juric, M. 2014, ApJ, 797, 70

Law, C. J., Bower, G. C., Burke-Spolaor, S., et al. 2015, ApJ, 807, 16

Lorimer, D. R., Bailes, M., McLaughlin, M. A., Narkevic, D. J., \& Crawford, F. 2007, Sci, 318, 777

Luan, J., \& Goldreich, P. 2014, ApJL, 785, L26

Masui, K., Lin, H., Sievers, J., et al. 2015, Natur, 528, 523

McLaughlin, M. A., Lyne, A. G., Lorimer, D. R., et al. 2006, Natur, 439, 817

Pen, U.-L., \& Connor, L. 2015, ApJ, 807, 179

Petroff, E., van Straten, W., Johnston, S., et al. 2014, ApJL, 789, L26

Petroff, E., Bailes, M., Barr, E. D., et al. 2015, MNRAS, 447, 246

Rane, A., Lorimer, D. R., Bates, S. D., et al. 2016, MNRAS, 455, 2207

Ravi, V., Shannon, R. M., \& Jameson, A. 2015, ApJL, 799, L5

Scholz, P., Spitler, L. G., Hessels, J. W. T., et al. 2016, arXiv:1603.08880

Siemion, A. P. V., Bower, G. C., Foster, G., et al. 2012, ApJ, 744, 109

Spitler, L. G., Scholz, P., Hessels, J. W. T., et al. 2016, Natur, 531, 202

Spitler, L. G., Cordes, J. M., Hessels, J. W., et al. 2014, ApJ, 790, 101

Thompson, D. R., Wagstaff, K. L., Brisken, W. F., et al. 2011, ApJ, 735, 98

Thornton, D., Stappers, B., Bailes, M., et al. 2013, Sci, 341, 53

Thornton, T. 2013, PhD thesis, Univ. Manchester

Trott, C. M., Tingay, S. J., Wayth, R. B., et al. 2013, ApJ, 767, 4

Vedantham, H. K., Ravi, V., Mooley, K., et al. 2016, arXiv:1606.06795

Wayth, R. B., Brisken, W. F., Deller, A. T., et al. 2011, ApJ, 735, 97

Wayth, R. B., Tingay, S. J., Deller, A. T., et al. 2012, ApJL, 753, L36

Williams, P. K. G., \& Berger, E. 2016, ApJL, 821, L22

Williams, P. K. G., Berger, E., \& Chornock, R. 2016, ATel, 8752 